\title{
Didier Moreau
}

\section{L'éthique professionnelle des enseignants : déontologie ou éthique appliquée de l'éducation?}

\section{Introduction}

L'acte d'enseigner semble être devenu plus difficile qu'il ne le fût jamais, non pas tant qu'il ait fondamentalement changé de signification ou d'horizon, ni non plus qu'il laisse techniquement démunis ceux qui l'exercent - lorsque les apports des recherches en éducation ouvrent des possibilités inouïes d'analyse et de compréhension de l'éducation scolaire, mais bien plutôt parce qu'il implique désormais une éthicité réfléchie de la part de ceux qui l'exercent, et que la source de cette éthicité est l'objet d'une tension importante entre les institutions et les acteurs : celles-ci demandent à ceux-là de concevoir éthiquement leur profession, ce à quoi les acteurs répondent par la demande adressée à l'institution de leur fournir et de leur rendre disponible cette éthique introuvable.

Nous proposons dans ce travail de clarifier la question de la source de l'éthicité propre à l'acte éducatif en général, en nous appuyant sur les résultats de recherches relatives à la construction de l'éthique professionnelle des enseignants au cours de leur formation et lors de leur entrée dans le métier. Nous préciserons comment ces recherches ont permis de poser l'hypothèse d'une éthique appliquée de l'éducation, et, à travers sa mise à l'épreuve dans le champ de la construction de la professionnalité enseignante, nous apporterons quelques éléments pouvant éclairer le débat entre la nécessité d'une déontologie de source institutionnelle, ou la possibilité de prendre confiance dans cet «homme capable », pour reprendre l'expression de Paul Ricœur, que peut devenir l'enseignant pour peu qu'il ait été formé en vue de l'imputabilité personnelle de son agir.

\section{a) Comment interpréter l'éthicité des enseignants ?}

La profession d'enseignant est donc confrontée à cette requête qui semble générale actuellement de devoir attester une éthicité propre. Il est en effet demandé aux jeunes enseignants de donner une dimension éthique à leur engagement professionnel, et aux plus anciens d'être aptes à entreprendre une réflexion morale accompagnant de manière critique l'exercice de leur métier. Cette injonction à l'éthique est plus qu'une mode passagère ou que l'expression d'un désarroi contemporain. Elle est portée par un mouvement de pensée que Michel Fabre a caractérisé comme un écho de la postmodernité en éducation ${ }^{1}$, et que nous avons analysé à travers le concept de post-historicité ${ }^{2}$. Quoiqu'il en soit, cette injonction à l'éthique peut être abordée à travers trois types d'interprétations.

Selon le premier, l'activité professionnelle spontanée des enseignants souffrirait de la même dérégulation que l'agir général de l'époque, et il serait alors nécessaire que l'institution de tutelle imposât de l'extérieur un cadre éthico-juridique apte à restaurer la régulation déficiente. Mais cette interprétation doit prendre en compte le processus de professionnalisation des enseignants : aussi, à la normativité et à la prescriptivité caractérisant l'encadrement des métiers, l'institution préfère désormais la régulation propre à la déontologie professionnelle : une injonction externe à construire dans la communauté elle-

\footnotetext{
${ }^{1}$ Fabre M. Le problème et l'épreuve : formation et modernité chez Jules Verne, Paris, Editions L'Harmattan, 2003.

${ }^{2}$ Moreau D. « Le recueil de soi à l'épreuve de la post-historicité », Horizons Philosophiques n¹ Vol. 15, 2004.
} 
même un code pour normer l'agir professionnel. Mais se pose visiblement la question d'une médiation capable d'assurer le passage du politique à l'éthique lorsque le juridique fait défaut, car, contrairement aux corporations dotées juridiquement d'une déontologie et d'une instance collégiale de reddition de comptes (Ordre des médecins, bâtonnier des avocats, etc.), le législateur en France n'a pas voulu étendre la professionnalité des enseignants au-delà d'une autonomie pédagogique limitée à l'herméneutique des programmes. Et on conçoit mal qu'une instance collégiale puisse s'élever contre un principe hiérarchique -même à l'Université. C'est pourquoi ce premier type d'interprétation, s'il était conduit schématiquement, aboutirait au paradoxe d'une déontologie impossible : moins contraignante qu'un code prescriptif, plus déroutante qu'une véritable autonomie morale, elle produirait les effets contraires à cette régulation éthique des pratiques enseignantes qu'elle recherchait. C'est ainsi qu'il est possible de comprendre le malaise exprimé par les jeunes enseignants du secondaire de manquer de cadres juridiques propres à orienter leurs responsabilités, et d'y voir l'origine de la loi du 15 Mars 2004 relative aux signes religieux ostensibles.

La deuxième interprétation est portée par la sociologie des valeurs ${ }^{3}$. Elle postule que les jeunes débutants détectent et reproduisent les traits éthiques propres aux acteurs appartenant à la corporation à laquelle ils s'intègrent. Mais cette interprétation laisse irrésolues des questions redoutables portant principalement sur l'origine de l'axiologie propre à tel groupe professionnel. Cette source est-elle justifiable par l'utilitarisme, comme la recherche du plus grand bénéfice pour les membres du groupe, ou par une approche conséquentialiste $^{4}$, comme choisir les valeurs faisant préférer les actions dont les conséquences sont cohérentes avec les buts supposés de la corporation ? Quoiqu'il en soit, la sociologie des valeurs ne peut pas analyser le poids moral propre que chaque nouveau professionnel apporte à sa communauté, et la force qu'il peut exercer, en tant que singularité, sur l'éthicité concrète de sa corporation.

Si la première interprétation plaçait la source de l'éthicité dans une instance externe, la seconde la dissout de telle sorte qu'elle se perde. C'est pourquoi nous avons privilégié dans nos recherches un troisième type d'interprétation, selon lequel l'enseignant est non seulement un acteur professionnel, mais également un agent moral.

Un acteur accomplit ses tâches à partir de l'interprétation qu'il fait du rôle qui est le sien, et cette interprétation du rôle est rendue possible grâce à des représentations. Mais l'éthique ne doit rien aux représentations ! On ne peut pas se représenter ce qui doit être; est objet de représentation seulement ce qui est accessible, précisément, à la connaissance. Le devoir-être, et c'est Aristote qui le caractérisa avec le plus de rigueur, est objet de délibération rationnelle. Un agent moral est ainsi, par définition, celui qui délibère relativement aux actes qu'il doit accomplir, selon le principe mis en évidence par Aristote pour distinguer la praxis de la techné qui exige que la qualité des moyens mis en œuvre soit homogène à la qualité de la fin visée. L'agent moral agit ainsi en veillant à chaque phase de son action à ce qu'elle reste intrinsèquement morale, de part en part orientée vers le meilleur but possible recherché. Cette interprétation cependant ne prétend pas que tous les enseignants soient factuellement des agents moraux, ou même que certains le soient constamment. Elle pose seulement qu'un enseignant professionnel peut l'être s'il prend en charge effectivement la tâche éducative corrélée à son agir professionnel. Comme on le saisit, cette interprétation vise l'horizon transcendantal de l'éthique de l'éducation, et réintroduit ainsi la précellence d'une méthode, celle d'une analyse des concepts, afin de préparer une étude empirique des faits. La source de cette analyse est simple, même si elle s'avère difficile : elle considère que l'acte éducatif

\footnotetext{
${ }^{3}$ Estrela M. T. Autorité et discipline à l'école, Paris, ESF, 1996.

${ }^{4}$ Pettit P. «Conséquentialisme et psychologie morale », Revue de Métaphysique et de Morale, n²/1994.
} 
possède une spécificité irréductible, et qu'il n'est pas possible de comprendre l'action de l'enseignant professionnel hors de cette spécificité éducative. Ce qui semble ainsi un truisme n'est pas cependant pris en compte dans nombre d'approches analogiques qui tendent curieusement à se généraliser.

La caractérisation éthique des activités professionnelles est profondément marquée en effet par une dichotomie, posée à l'origine par la pensée grecque, attestée chez Homère, reprise par la réflexion anglo-saxonne et que Lemosse ${ }^{5}$ a contribué, avec d'autres, à introduire dans le champ francophone. Cette dichotomie oppose les professions d'orientation altruiste aux métiers exercés pour l'intérêt premier de l'artisan et susceptibles de répondre factuellement - à l'utilité d'autres personnes. Partant, il était tentant de fonder, à l'intérieur même de l'ensemble des professions, des classes définies par le type d'orientation altruiste qui y est engagé. C'est ainsi que le concept de caring a été introduit, rassemblant les professions fondées sur ce souci d'autrui, comme les professions de santé et les professions de l'enseignement et de l'éducation ${ }^{6}$. Or nous pensons fortement que cette catégorisation aboutit au résultat contraire à son principe même, et que, loin d'apporter une compréhension nouvelle, elle est la cause d'un obscurcissement de la question de l'éthique dans le champ de l'éducation. Il est possible de reconnaître que la relation pédagogique n'est en rien une relation thérapeutique, sauf à prendre certaines métaphores relatives à la "guérison de l'ignorance » au pied de la lettre, et il est nécessaire de penser qu'autrui, tel qu'il est présent dans l'acte éducatif, n'est pas une individualité comme l'est un patient par exemple.

L'interprétation de l'éthicité des enseignants que nous privilégions ainsi part de l'hypothèse, peu répandue il est vrai, d'une spécificité intrinsèque de l'acte éducatif : c'est parce que l'acte éducatif est structuré a priori comme un acte éthique, qu'il peut être $a$ posteriori investi par un projet moral porté par celui qui l'accomplit : l'acteur professionnel peut devenir un agent moral parce qu'il comprend qu'il le doit, s'il veut éduquer, et l'éthique ne peut plus se présenter alors comme un «supplément d'âme » dont on voudrait que les enseignants soient dotés, pour le bien de la communauté. Ce qui, on peut le saisir dès à présent, n'oblige ni un professionnel de la santé, ni un conseil juridique qui, pour cette raison même, ont besoin d'une déontologie. Or, quel est le fondement de cet apriorisme que nous mettons en avant ? Ce n'est pas le simple refus du positivisme empirique qui nous y mène, et pas davantage un arrière-plan prescriptif masqué - un moralisme préconçu. C'est, une fois de plus, l'analyse philosophique des concepts. Nous pensons en effet que la tâche présente de la philosophie de l'éducation n'est plus tant de fournir en catégories, en notions et en concepts le marché des idées, mais d'aider à comprendre et à interpréter les phénomènes de l'éducation, au-delà d'une fallacieuse causalité dont l'explication positiviste a abondamment recouvert le réel, au point de le rendre méconnaissable. Et singulièrement, le champ de l'éthique.

\section{b) La structure éthique de l'éducation.}

Qui est cet autrui qui est concerné par l'éducation? Il est d'abord ce que les grecs nommaient les Neö̈, et que nous avons bien du mal, désormais à désigner. Les Neoï, c'est cet autrui-nouveau qui advient à toute communauté à condition qu'elle accepte de ne pas le

\footnotetext{
${ }^{5}$ Lemosse M. «Le professionnalisme des enseignants : le point de vue anglais »; Recherche et formation n6/1989.

${ }^{6}$ Noddings N. Caring, a feminine approach to Ethic and moral education, Los Angeles, Univ. California Press, 1984.
} 
réduire à ce qui est déjà présent, mais qu'elle le considère comme chance ${ }^{7}$; les Neö̈ commandent ainsi un mode particulier du respect, non pas en raison de leurs déficits ou de leurs incapacités - ce qui sera le propre de la pensée moderne, mais parce qu'ils promettent un enrichissement de l'interprétation du monde, un «rajeunissement» du sens de notre expérience (les romains désignaient les Neö̈ par le terme de juventes). Mais en tant que promesse, autrui-nouveau ne peut être respecté que par l'entreprise d'une éducation, parce qu'il est inachevé - non en tant qu'individu, ce qui n'a pas de sens, car nous sommes tous des êtres de finitude, mais en tant qu'interprète et acteur futur du monde. L'éducation est ainsi la tâche éthique essentielle que nous devons entreprendre vis-à-vis des Neoï. C'est le fondement même du droit à l'éducation ${ }^{8}$, souvent obscurci parce que les Neö̈, en tant que structure d'existence, sont rabattus sur la subjectivité, concrète et privée, d'élèves dans l'état juridique de minorité.

Mais autrui est également représenté par les Neö̈ des Neö̈, c'est-à-dire que l'éducation vise un horizon qui dépasse la proximité de ceux qui nous succèdent: nous voulons qu'à leur tour «ceux que nous laisserons après nous », pour reprendre l'expression d'Aristote, puissent éduquer leurs propres successeurs, dans la fidélité au principe de respect qui nous a conduit à les éduquer eux-mêmes. Autrui dans l'éducation est un horizon dont on voit bien qu'il se dérobe sans cesse: on peut nommer cela, avec Hans Jonas 9 , les « générations futures », ce dont il s'agit est cette prise en compte de l'inachèvement du sens, de nous-mêmes et du refus de l'immanence absolue qui consisterait à ce qu'une génération se présentât un jour comme l'humanité enfin réalisée. L'éducation est éthique en ce qu'elle refuse ce totalitarisme d'une détermination achevée de l'essence de l'homme, selon un modèle à réaliser. Ce qu'il y avait de paradoxal et d'absurde dans les thématiques totalitaires de l'Homme Nouveau, c'est que précisément les Neö̈ n'y avait pas leur place, écrasés par le poids des exigences de l'idéologie.

Au respect dû aux Neoï se superpose donc le respect dû au monde qu'ils pourront construire, si la possibilité leur en est donnée par l'éducation. L'éducation est donc la formulation du droit qu'ont les générations futures à exister et à déterminer de manière responsable le monde qui sera le leur. De ce point de vue, elle vise à l'auto-affirmation d'autrui. Autrui, dans l'éducation, ne se rencontre pas comme individu, et c'est le premier paradoxe de l'éducation, qui en fait comme l'a dit Kant le premier, une tâche impossible. Car l'éducateur, l'enseignant, ont un rapport avec des individualités. Or le premier caractère des Neoï est d'être-en-devenir. Et c'est ce que disent les pédagogues, de plutôt s'attacher chez l'élève à la promesse d'un développement, qu'à la déception d'une insuffisance actuelle. Mais l'être-en-devenir ne s'accomplit véritablement que s'il est mis en rapport avec la culture et le savoir de la communauté humaine - jamais l'éducation, par définition, n'a pensé ce devenir comme un processus d'auto-développement de l'individu. C'est dans l'œuvre pédagogique de Schleiermacher que l'on rencontre cette thématique posée le plus lucidement : l'éducation vise à transformer l'individualité en singularité, par la mise en relation avec la communauté

\footnotetext{
${ }^{7}$ Moreau D. «L'insertion dans l'être : la question de l'éducation dans la philosophie de Merleau-Ponty »; Penser l'éducation, $\mathrm{n}^{\circ} 17,2005$.

${ }^{8}$ Moreau D. «Le droit à l'éducation : un enjeu de l'éthique appliquée des enseignants professionnels »; Le droit à l'éducation : quelles effectivités du Nord au Sud? Actes du Colloque AFEC de Ouagadougou, Mars 2004.

${ }^{9}$ Jonas H. Pour une éthique du futur, Paris, Payot, 1998. La difficulté du concept chez Jonas est qu'il est chargé de la signification onto-théologique selon laquelle nous avons la charge de l'être-à-venir parce qu'il nous est assigné par notre origine-même, totalement transcendante : les «générations futures » manifestent notre essence et indiquent la possibilité de notre salut.
} 
humaine, par la culture et les savoirs. Et seule la singularité de chacun peut enrichir notre expérience commune du monde ${ }^{10}$.

C'est pourquoi la structure éthique de l'acte éducatif se révèle si spécifique : aucune autre activité humaine, même explicitement orientée vers autrui, ne rencontre l'humanité sur un mode aussi fondamental, et c'est ce qui justifie, à nos yeux, le caractère essentiel de l'approche philosophique en éducation. Mais la tâche de la philosophie n'est pas de collecter ou d'enregistrer des événements, elle est plutôt d'aider à les penser et à les comprendre. Aussi, toute recherche visant à interpréter l'éthicité d'hommes au travail, si elle commence par l'élucidation des concepts, doit-elle cependant se poursuivre aussitôt par une enquête empirique sur les actes et discours de ceux qui sont concernés.

\section{c) Problématique d'une recherche sur l'éthicité des enseignants.}

Lorsque nous avons engagé notre travail pour connaître l'origine de l'éthicité des enseignants professionnels, nous avons privilégié la notion de position d'agent moral, en la distinguant bien des autres approches relatives aux représentations et aux postures professionnelles. Etre ou devenir un agent moral, c'est prendre position, par ses actes et ses paroles, quant à la fin éthique poursuivie par ceux-ci, c'est tenter d'assumer cette cohérence fondamentale qui, selon Aristote, définit la praxis. A chaque moment donc, une position morale est objet de délibération et de réflexion: elle n'est pas source habituelle des comportements, comme pourrait l'être une représentation sur la moralité, ou une posture acquise par intériorisation de patterns. On peut même affirmer qu'il n'y a que la position morale qui exige cet effort rationnel intégral.

L'hypothèse de notre recherche a donc été, dès l'origine, que les enseignants qui débutaient dans la profession se trouvaient confrontés à cette exigence - portée par la structure même de l'acte éducatif, d'avoir à conquérir une telle position d'agent moral, et qu'ils avaient le choix, parfaitement libre, de ne pas s'y engager. Une position d'agent moral en effet n'oblige pas au-delà d'une cognition rationnelle, et reconnaître sa portée rationnelle ne comporte aucune motivation à s'y atteler. Ainsi, ce n'est pas parce qu'un acteur reconnaîtra qu'il est préférable, relativement au sens même de l'acte éducatif, d'y agir moralement, qu'il se sentira contraint par cette cognition, et il pourra décider pour des raisons purement subjectives de l'ignorer totalement. C'est la supériorité de la notion de position morale que d'éviter de recourir à des arrière-plans qui ne soient pas accessibles phénoménologiquement, comme la Lebensform, la Forme de Vie à laquelle le sujet a appartenu avant son entrée dans le métier d'enseignant. Ce n'est pas dire, bien évidemment, que de telles enquêtes soient dénuées d'intérêt, elles sont d'autant plus nécessaires qu'elles seules permettent de mieux saisir la motivation à agir qui doit succéder à la cognition pour que l'acte moral soit entrepris, et d'analyser comment cette motivation prend sa source dans les valeurs propres à la Lebensform du sujet. Mais l'erreur la plus communément commise par les recherches positivistes est de penser que les valeurs peuvent être à la fois la causa efficiens et la causa finalis de l'éthicité concrète. Nous pensons tout au contraire que les valeurs ne sont que la causa materialis de l'agir moral. La cause finale étant la recherche délibérée de l'horizon transcendantal de l'essence de l'éducation et la cause formelle, pour être fidèle à la doctrine aristotélicienne des quatre causes, étant à rechercher dans la réflexion et la distanciation consécutive à l'épreuve de la réalité, ici : l'expérience professionnelle. Quant à la cause efficiente, il faut tenir comme étant nécessaire qu'elle est le résultat de la délibération

\footnotetext{
${ }^{10}$ Moreau D. «Jean-Paul, lecteur de Jean-Jacques ; l'approche herméneutique de l'éducation », Le Télémaque, $\mathrm{n}^{\circ} 27$.
} 
morale elle-même, c'est-à-dire la décision de l'agent moral. Toute autre détermination invalidant ipso facto le caractère moral de l'action.

On le voit donc, la notion de position morale permet seule de rendre compte de manière éthique de l'éthicité d'acteurs humains. Quel serait en effet le sens éthique d'une recherche en éducation qui ferait l'hypothèse que les acteurs ne sont pas libres de leurs choix moraux, qu'ils n'obéissent qu'à des représentations, des désirs ou des fantasmes ? Elle ne pourrait pas longuement cacher ses arrière-pensées prescriptives ou coercitives, et en aucun cas elle ne serait respectueuse des acteurs sur lesquels elle prétendrait travailler; en un mot, ce ne serait pas une recherche déontologiquement acceptable...Si nous nous en convainquons, en revanche, il nous faut trouver un moyen -cohérent avec les fins recherchées, pour entrer en contact avec les positions éthiques des enseignants. Et c'est ici que la double face de la métaphore de la position va se révéler féconde. Une position éthique est d'abord une position stratégique qu'il faut d'abord conquérir puis occuper; elle est de nature nécessairement conflictuelle, car elle va se heurter à des croyances, à des préférences et à des habitudes personnelles de l'acteur : elle va imposer des choix et des renoncements. Il va falloir ensuite la défendre, mais comment? L'agent qui conquiert sa position éthique est un stratège, mais non un tacticien, car il lui manque le savoir-faire de l'expérience, et le désarroi rend amère la victoire. Enfin, une prise de position est un acte public qui rend visible et qui expose, c'est la deuxième face de la métaphore. L'agent ne peut plus s'appuyer sur des convictions morales élaborées in foro interno dans la mesure où il accède à la pleine visibilité éthique, requise par son exercice professionnel qui cesse d'être un agir de la sphère privée. S'il peut se conduire comme bon lui semble dans sa vie personnelle et n'avoir de comptes à rendre qu'à sa conscience morale - car dans la sphère privée, l'inconduite est plutôt interprétée du point de vue des affects que de la mauvaise délibération, en revanche l'exercice d'une profession exige la reddition de comptes de celui qui n'est plus sujet mais acteur d'un rôle qui lui est confié. C'est l'origine de toute déontologie professionnelle : interdire que l'on puisse exercer une charge, un rôle ou une fonction avec la désinvolture et l'insouciance qui, parfois, sont la tonalité majeure de la vie personnelle.

La position éthique est délibérée et publique, elle est donc accessible à la recherche. Mais comment la recueillir? la démarche que nous avons choisie fut celle de l'herméneutique, conduite méthodologiquement par l'intercompréhension des positions éthiques dans des entretiens semi-dirigés, dont les propositions furent analysés suivant leur direction illocutoire.

\section{d) L'hypothèse d'une éthique appliquée.}

Les premiers résultats orientèrent l'interprétation vers l'idée d'une structuration de l'éthicité professionnelle ${ }^{11}$. Les positions éthiques ne pouvaient être tenues qu'en cohérence entre elles, contrairement à des valeurs qui peuvent s'avérer conflictuelles. C'est-à-dire que les positions fonctionnent comme les propositions d'une axiomatique, comme l'avait montré $\mathrm{J}$. Vuillemin à propos des systèmes éthiques ${ }^{12}$ : une proposition excluant, par le choix qui en avait été fait, une autre proposition mais en imposant en revanche une tierce ; chaque décision morale orientant systématiquement les autres propositions formulées sur l'éthique. Là où la sociologie ne pouvait collecter que des faits rhapsodiques, nous avons pu mettre en évidence la systématicité et la structuralité de l'éthique des enseignants, corrélats nécessaires et donc probants de son caractère réflexif et rationnel. Mais l'autre aspect de cette systématicité est

\footnotetext{
${ }^{11}$ Moreau D. « La construction de l'éthique professionnelle des enseignants : la genèse d'une éthique appliquée de l'éducation »; Thèse de Doctorat sous la direction de M. Altet, Université de Nantes, Février 2003.

${ }^{12}$ Vuillemin J. Nécessité ou contingence, Paris, Minuit, 1984.
} 
qu'elle résulte d'un processus dynamique, qui nous a permis alors de soutenir l'idée d'une genèse de l'éthique professionnelle, conduite à travers un processus de construction de l'éthicité de l'agent : l'acteur professionnel devient un agent moral, en s'engageant dans un procès d'élaboration de structures éthiques capables d'orienter son agir.

Mais pour quoi faire ? Loin d'octroyer un «supplément d'âme », comme Bergson le demandait à la morale pour corriger l'aveuglement de la technique, ou d'être une justification a posteriori habillant habilement une errance pratique, l'éthique professionnelle des enseignants est apparue comme le résultat de la résolution de problèmes liés à l'action concrète et quotidienne, loin des déclarations principielles et abstraites. C'est parce qu'ils se trouvent confrontés à des problèmes éthiquement valides et dont les solutions techniques s'avères insuffisantes, que les enseignants débutants peuvent s'engager dans la recherche de structures et de catégories leur permettant d'envisager des solutions. C'est parce qu'ils se trouvent insatisfaits de leur manière spontanée d'agir qu'ils vont tenter, par la délibération rationnelle, de produire des normes valides capables de guider leur futures décisions. En un mot, nos recherches ont posé l'hypothèse que l'éthique des enseignants professionnels possédait les caractères d'une éthique appliquée.

Le concept même d' «éthique appliquée » souffre en France, nous l'avons souvent constaté, de nombreuses confusions. Certains l'entendent comme une sub-éthique, d'autres encore -un peu mieux informés, n'acceptent pas d'y voir autre chose qu'une néo-casuistique. A l'origine de toute éthique appliquée il y a l'affirmation de la spécificité de l'Application, telle que Gadamer la formule ${ }^{13}$. Dans les théories morales classiques de la modernité, la connaissance des principes permet de dégager à coup sûr les maximes de l'action à conduire, et l'application n'y est considérée que comme une déduction, faisant passer de l'universel au particulier, comme chez Kant. Ces théories mettent en avant l'idée d'une éthique centrée sur la conviction morale du sujet et sur la perfection de ses intentions. Mais qu'advient-il, si les garanties métaphysiques d'un Savoir absolu, d'un Regard Omniscient (ce que Thomas Nagel appelle le «point de vue de nulle part ${ }^{14}$ viennent à manquer?

Dans les perspectives postmodernes en effet, le savoir est une construction fragile et menacée, qui suppose que s'établisse la mutuelle compréhension de ceux qui y participent ; or le partage des expériences montre au contraire que, selon la formule de Schleiermacher ${ }^{15}$ c'est plutôt la mécompréhension qui est la règle, et la parfaite compréhension l'exception. La raison en est à rechercher dans cette attitude fondamentale de l'homme qui est l'interprétation, en vue de la construction d'un sens. Contrairement à ce que voulait la pensée moderne, l'homme n'est pas face à des objets signifiants par eux-mêmes, car le sens, lui, n'est pas un objet du monde, facilement accessible à la pensée, comme le seraient ces objets physiques. Le sens est toujours à venir, incertain, éphémère, sans cesse à la recherche d'un nouvel enracinement dans la confirmation d'un partage. Toute lecture, toute discussion, tout effort de connaissance, sont des tentatives de construire un sens par l'interprétation, et aucune interprétation ne peut être validée par un principe qui lui serait extérieur. Nous ne pouvons pas sauter hors du cercle interprétatif. C'est le fondement de l'herméneutique philosophique, telle que Gadamer la définit, à partir de Schleiermacher, Dilthey et Heidegger. Il en résulte dans le domaine moral qu'aucune action ne peut être garantie avec certitude a priori comme étant moralement bonne, et que l'application devient alors une phase tout à fait originale de la délibération morale : l'application devient elle-même créatrice de normes.

Parallèlement à l'herméneutique philosophique, les analyses de Max Weber ${ }^{16}$ ont permis de dépasser l'aporie du post-kantisme. Dans le monde contemporain complexe, il n'est

\footnotetext{
${ }^{13}$ Gadamer H. G (1996) ; Vérité et méthode, Seuil, Paris.

${ }^{14}$ Nagel T. Le point de vue de nulle part, Combas, L'éclat, 1993.

${ }^{15}$ Schleiermacher F. L'Herméneutique de 1801, Paris, Cerf, 1987.

${ }^{16}$ Weber M. Le savant et le politique, Paris, UGE, 1963.
} 
pas possible de connaître avec précision et certitude quelles seront les conséquences de chaque action : les interdépendances entre les acteurs et les sphères multiples de l'agir sont désormais hors de portée d'une pure conscience réfléchissante. Weber analyse les conséquences de cette complexité dans le monde moral et montre qu'elle réinterroge la question de la responsabilité. Il oppose alors une éthique de la conviction, selon laquelle l'agent se détermine à partir de ce qu'il tient pour juste et pour bon, et se satisfait in foro interno de l'excellence morale de ses maximes, à une éthique de la responsabilité dans laquelle l'agent envisage les conséquences probables de ses actions pour arrêter ses décisions. Dans une éthique de la responsabilité, le problème de l'application passe ainsi au premier plan : que signifie en effet la validité éthique d'un principe si son application aveugle produit des effets catastrophiques?

C'est dans ce constat que l'éthique appliquée puise sa légitimité. Le développement des activités s'appuyant sur la science et la technique nous amène à prendre des décisions dont nous ne pouvons connaître avec certitude les effets pratiques et les conséquences morales. Le paradoxe est donc bien d'être responsable dans l'incertitude, c'est-à-dire, d'être responsable de l'incertitude. Ainsi commence l'éthique herméneutique: prendre en charge l'agir contemporain qui creuse son sillon dans l'inconnu parce que se sont perdues les voies balisées du progrès émancipateur. Nous devons à Kurt Bayertz, Professeur à l'université de Münster, d'avoir défini le premier paradigme d'une éthique appliquée ${ }^{17}$. En posant l'alternative de savoir «si l'humanité subit l'évolution sociale comme une force de la nature et la lie à elle ou si cette évolution peut être dirigée selon des critères rationnels et humains », il conclut : «Actuellement, peu de choses donnent à penser que ce problème puisse être résolu, théoriquement comme pratiquement ${ }^{18}$. $\gg \mathrm{Ni}$ la générosité de la Providence, ni sa sécularisation dans l'achèvement de la Raison ne peuvent plus apparaître comme des paradigmes interprétatifs aptes à éclairer nos choix, et sans doute convient-il de répudier l'alternative elle-même. L'année 1992 est la date choisie par K. Bayertz de la reconnaissance de l'éthique appliquée dans l'université allemande à travers la publication de l'Almanach de l'éthique pratique par Meggle, Rippe et Fehige, qui recensa les travaux appartenant à ce champ spécifique et qui se situaient dans la voie ouverte aux Etats-Unis par Peter Singer ${ }^{19}$.

Cette éthique pratique n'est pas une casuistique en ce qu'elle ne traite pas de cas individuels, d'agents moraux dans des situations toujours singulières, mais au contraire de problèmes ayant une signification générale, tels ceux posés par les développements de la biologie contemporaine, la complexification de la justice distributive, l'accélération de la pression des activités humaines sur l'environnement. Contrairement à la casuistique, dans laquelle la particularité des cas interdit toute construction de savoirs, contrairement également aux expériences de pensée comme Kant lui-même les proposait en vue d'en subsumer des principes, l'éthique appliquée se concentre sur des problèmes réels qui ont une portée générale et qui présentent ainsi un intérêt public, même s'il ne s'étend pas au-delà du groupe professionnel des acteurs qui s'y trouvent confrontés. L'éthique appliquée ne vise pas universellement et abstraitement «tout être doué de raison » mais précisément des hommes dont les actions rencontrent un obstacle éthiquement (et non techniquement) déterminé. Si ces problèmes peuvent intéresser rationnellement tout agent moral quel qu'il soit, il n'en demeure pas moins qu'ils ne se posent factuellement qu'à certains acteurs, et que c'est cette rencontre même qui définit éthiquement les enjeux et tensions propres à telle activité professionnelle.

Aussi, contrairement à la casuistique, l'éthique appliquée peut poser qu'il y a une solution générale à une classe de problèmes et qu'elle est accessible par une discussion

\footnotetext{
${ }^{17}$ Bayertz K. «Qu'est-ce l'éthique appliquée ?»in : La vie des normes \& l'esprit des lois, L. Sosoe dir., Montréal, Harmattan, 1998.

${ }^{18}$ Bayertz K. « Y a-t-il des limites éthiques à la recherche scientifique? »Colloque de Neuchâtel, octobre 1997.

${ }^{19}$ Singer P. Practical ethics, New York, Cambridge Univ. Press, 1993.
} 
rationnelle. La casuistique ${ }^{20}$ refusait qu'il existât une telle issue, car la rechercher conduisait assurément à l'aporie : seule peut être envisagée une réponse à une situation particulière, non généralisable à d'autres possédant des traits voisins. L'éthique appliquée sépare ainsi deux questions : celle de l'examen de la validité des principes, et celle de leur application cohérente et pertinente. A partir de ces deux questions, K. Bayertz distingue trois groupes de problèmes moraux.

- Le premier est celui qui résulte de la collision de principes : dans une situation donnée, deux principes éthiques fondamentaux peuvent également être invoqués pour produire une norme d'action, mais leurs issues respectives sont contradictoires : solidarité/égalité, par exemple ( cf. le problème de la discrimination positive dans l'institution scolaire).

- Le second est la conséquence d'un manque de clarté dans la problématisation morale de faits jugés empiriquement pertinents : comment remonter aux principes pour interpréter moralement une situation complexe?

- Le troisième est lié à la complexité de l'agir : ce sont les problèmes issus de conflits entre motivations morales et mobiles extra-moraux : comment traduire en acte ce que l'on pense être juste, ou inversement, comment orienter moralement ce qui apparaît pragmatiquement nécessaire ?

Ce que l'éthique appliquée permet d'éviter, dans ces classes de problèmes, est l'apparition de ce qui chez Kant était présenté comme conflit des devoirs, et qui comme tel était réfuté dans la mesure où toute conscience peut, dans un acte simple d'examen et de réflexion, reconnaître son devoir. Ou, ce qui serait le résultat de l'application d'une déontologie externe, la constitution d'une hiérarchie des principes (l'égalité avant la solidarité), qui ne serait, in fine, qu'un système relativiste de valeurs, s'opposant dogmatiquement aux systèmes concurrentiels. Nous reviendrons sur cette limite.

Face à ces classes de problèmes, l'éthique appliquée propose une démarche cohérente dont la mise en œuvre commence par une analyse explicative des dilemmes, conflits et collisions de principes et qui posant l'hypothèse d'une décision concevable, évalue le prix à payer qui en résultera. Cette phase évaluative correspond effectivement à cet exercice de la responsabilité qui caractérise l'abandon d'une éthique de la conviction. Enfin, le recueil des décisions acceptables dans la portée de ce qui est prévisible permet la production de normes en vue d'actions futures. L'éthique appliquée fait donc, comme le demandait Gadamer, de l'Application un moment herméneutique permettant de comprendre comment un principe abstrait est toujours valide pour éclairer une situation nouvelle, ce qui coïncide avec ce que Dworkin nomme l'Interprétation créatrice ${ }^{21}$.

\section{e) La nature de l'éthique professionnelle des enseignants.}

L'hypothèse directrice d'une éthique appliquée nous a alors amenés à privilégier l'attention portée sur la genèse même de l'éthique des enseignants et nous a conduits à constituer un corpus d'entretiens de Professeurs des Ecoles débutants d'ancienneté réduite échelonnée sur trois ans: PE2, titulaires première et deuxième année. Nous lui avons fait correspondre d'autres données, recueillies auprès d'enseignants confirmés ayant choisi de devenir formateurs de leurs jeunes collègues, dans la perspective de comprendre comment se réalisait l'étayage de cette genèse. Les résultats obtenus ont été de deux ordres : tout d'abord structurels, ce qui répondait bien à nos hypothèses, mais également événementiels, ce qui relevait plutôt de la sérendipité, de la découverte inattendue.

\footnotetext{
${ }^{20}$ Jonsen \& Toulmin (1989) ; The abuse of casuistry, Berkeley, Univ. of California Press, 1989.

${ }^{21}$ Dworkin R. L'empire du droit, Paris, PUF, 1994.
} 
Les enseignants débutants sont confrontés dès leur prise de fonction à des difficultés que la plupart d'entre eux interprètent comme relevant de l'éthique, et qui se présentent comme des dilemmes ou des apories. Ils découvrent alors que leurs convictions morales initiales sont insuffisantes pour les résoudre et qu'ils doivent faire face, par eux-mêmes, à des situations fort complexes qui outrepassent celles qu'ils avaient pu imaginer. Quelques-uns déplorent alors un manque de formation, d'autres enfin refusent toute épaisseur morale à des difficultés qu'ils traiteront plutôt techniquement, mais la plus grande part acceptera d'engager une problématisation éthique, en construisant une position éthique. Le point de départ est une éthique juridique, qui, partant du projet de construire des règles morales pour éclairer le comportement de chacun, peut se développer en un véritable cadre en vue d'organiser une vita studiosa $a^{22}$ propre à la classe. C'est la première structure de l'éthique professionnelle qui présente un effort de systématicité et de cohérence, puisque l'agir de l'enseignant y est nécessairement interrogé. Mais ce système ne concerne que les problèmes liés à la relation pédagogique propre à la classe conçue comme communauté d'apprentissage. Elle laisse le débutant démuni face à un autre ordre de conflits qui, cette fois, le cernent dans sa professionnalité même.

Si le débutant se représente généralement la difficulté de l'acte d'enseigner, il ne peut en aucun cas anticiper l'extrême diversité des questions éthiques que sa fonction d'acteur professionnel lui fera rencontrer, avec les élèves, les parents, les collègues, tout partenaire qu'il sera amené à rencontrer. Il réalise alors que cette dimension humaine de sa profession revendique une éthique surérogatoire, dépassant toute déontologie formelle qui aurait pu lui être présentée, et qu'il n'a pas les ressources suffisantes pour y parvenir. C'est pourquoi il s'oriente vers l'idée d'une éthique de la discussion, destinée à promouvoir autour de lui, dans l'école et entre collègues concernés en dehors de celle-ci, de véritables communautés communicationnelles ${ }^{23}$. Cette structuration correspond à l'abandon véritable de toute éthique de la conviction conçue in foro interno, et se traduit par la demande très forte des jeunes enseignants de former des groupes de discussion dans lesquels il soit possible de résoudre des problèmes éthiques. C'est ici que commence véritablement l'éthique professionnelle : dans l'abandon d'une instance monologique au profit d'un cadre dialogique en vue de la résolution de problèmes et de la création de normes.

Enfin, s'ébauche la constitution d'un troisième système éthique, dans l'effacement progressif du caractère rigide de l'éthique juridique et grâce aux premiers résultats obtenus dans la participation aux communautés communicationnelles. Il s'agit d'une véritable éthique herméneutique, qui fait passer au premier plan les exigences morales de l'acte d'éduquer, et qui s'attelle à la tâche, qui paraît infinie mais nécessaire, de travailler à la compréhension mutuelle qui permettra que chacun reçoive de l'éducation la possibilité de s'auto-affirmer comme sujet dans un monde dont il partagera le sens. Comme le dit Gadamer, comprendre est un cas particulier de l'application d'une généralité à une situation concrète. La poursuite de nos travaux nous a conduit à penser que c'est dans cette herméneutique que réside le noyau propre de l'éthique des enseignants, qui n'est pas commun aux autres éthiques professionnelles. Car c'est la source de la sérendipité, le surgissement du factum pédagogique dans son caractère singulier.

\section{f) Les épreuves herméneutiques de la construction de l'éthique des enseignants.}

\footnotetext{
${ }^{22}$ Expression en référence, bien sûr, à l'ouvrage de Hannah Arendt, Vita activa, Stuttgart / Berlin, Kohlhammer, 1960 .

${ }^{23}$ Habermas J. De l'éthique de la discussion, Paris, Cerf, 1992 ; Apel K. O. Ethique de la discussion, Paris, PUF, 1994.
} 
Dès les premiers entretiens en effet il est apparu que la construction de structures d'éthicité était rythmée par des événements, véritables scansions dans un processus dynamique. Ces événements étaient vécus comme des épreuves par les jeunes enseignants, auxquelles tous se trouvaient confrontés, mais auxquelles ils réservaient cependant un accueil personnel, en leur octroyant une valeur et un sens propres. A la lumière des recherches ultérieures ces épreuves sont apparues multiples, mais leur efficience identique : provoquer une conversion du regard, une éducation morale de soi au sens platonicien, ou bien, dans un sens plus contemporain, initier la construction de compétences interprétatives, herméneutiques plus précisément. Trois épreuves éthiques ont été ainsi discernées et décrites.

La plus éclatante est celle de la Vulnérabilité ${ }^{24}$. Cette épreuve peut être une attitude délibérée du débutant. Elle se passe alors en deux phases successives. Dans la première, l'enseignant découvre la vulnérabilité d'autrui : élève mais aussi parent, que son inexpérience met en difficulté. Son pouvoir insoupçonné peut être dangereux et autrui - élève y est exposé sans défense. Il lui importe alors de développer des compétences pour protéger autrui. Mais pour y parvenir, et c'est la seconde phase, le débutant doit lui-même s'exposer, vulnérable, afin qu'autrui en retour puisse se sentir protégé : ce n'est qu'en s'impliquant, en se mettant à $\mathrm{nu}$, en prenant des risques, que l'enseignant pourra assumer sa responsabilité vis-à-vis des plus faibles que sont - individuellement, les élèves. L'épreuve de la vulnérabilité ouvre alors la parole, non plus la parole magistrale, mais un dialogue où la finitude de chacun devient le ciment la communauté. La vulnérabilité fonde l'intercompréhension. On peut saisir aussi que, faute de ce projet de s'exposer à autrui, la relation pédagogique est vécue comme un affrontement permanent dont il s'agit de sortir victorieux par des stratégies de succès technique et de domination par le verbe. Le projet éthique s'est mué alors en projet politique. En revanche, si le débutant assume l'épreuve où il devient vulnérable, il permet que les élèves, en tant que sujets éthiques, construisent eux-mêmes des compétences grâce auxquelles chacun découvre qu'il doit répondre de l'autre afin de préserver le monde de la vie dans lequel il se sait lui-même protégé. Nous sommes ici aux antipodes d'une éthique monologique de la compassion.

Mais cette épreuve de la vulnérabilité, quoiqu'elle apparaisse phénoménologiquement première dans la recherche, n'est cependant pas dissociable dans l'expérience vécue d'une autre épreuve herméneutique - peut-être première dans l'ontogenèse, celle de l'opacité. En effet, très tôt est apparu qu'un certain type de problèmes catalysait en quelque sorte le moment de la vulnérabilité, et qu'il était centré sur la question des élèves en difficulté ${ }^{25}$. Un élève mis en difficulté à l'école est un alter incompréhensible, dont l'enseignant ne peut pas interpréter de manière fiable les actions, les attitudes, les représentations ou les croyances. Cette opacité peut avoir une source culturelle, et c'est d'abord ce qu'imagine le jeune débutant, avant de comprendre qu'elle n'est que la manifestation de l'énigme nécessaire que pose un sujet souffrant dont on ne peut saisir la démarche cognitive. Le premier résultat de cette épreuve est la destruction du fantasme de l'omnipotence pédagogique, et les débutants ne peuvent se réfugier très longtemps dans cette pensée magique qu'il suffit d'apporter à l'élève ce qui lui manque, pour venir à bout de son incompréhension. Car la grande difficulté de certains élèves ne laisse aucune prise à des solutions techniques. Si la vulnérabilité dévoile l'altérité, l'opacité révèle la singularité d'autrui: car ce qui n'est pas compréhensible dans l'élève en difficulté, ce n'est pas sa difficulté mais plutôt sa propre incompréhension de ce qui semble à l'expert transparent. Et dès lors, l'enseignant s'aperçoit qu'aucun élève n'est

\footnotetext{
${ }^{24}$ Moreau D. «L'épreuve de la vulnérabilité : une source de l'éthique professionnelle des enseignants »; Penser l'éducation $\mathrm{n}^{\circ} 14,2004$.

${ }^{25}$ Moreau D. «L'entrée dans le métier d'enseignant : les deux épreuves éthiques fondamentales », $7^{\text {ème }}$ Biennale de l'Education et de la formation, Lyon, 2004.
} 
compréhensible, parce qu'autrui n'est pas accessible, sauf dans l'illusion pédagogique dont le paradigme majeur est construit par Jean Itard instruisant Victor de l'Aveyron ${ }^{26}$.

Lorsque cette illusion tombe, lorsque se manifeste ce principe d'une incomplétude pédagogique, le risque est grand que le débutant ne se réfugie dans un scepticisme prononcé vis-à-vis des compétences des plus expérimentés comme pour les préconisations apportées par la réflexion pédagogique. Et cette attitude est assez légitime, dans la mesure où le noyau de la découverte est bien cette impossibilité du comprendre, marque de la finitude humaine. Aussi est-il nécessaire, décide alors le débutant, d'entreprendre un travail sur le déficit herméneutique, dont le but ne sera plus la compréhension parfaite, la transparence radicale, mais une approche respectueuse de la singularité d'autrui, afin de limiter graduellement les effets de la mécompréhension. La modification du projet éthique qui en résulte pour l'enseignant est radicale : il ne s'agit plus d'apporter à un être déficient ce qui lui manque en matière de savoirs ou d'habiletés, mais de l'aider à ce qu'il nous aide à le comprendre. C'est parce que les hommes se comprennent mal, comme l'avait montré Schleiermacher, qu'ils coopèrent et qu'ils dialoguent. Ce que les enseignants installent alors dans leur pratique de classe est un intermonde de traduction, un espace mutuel de dialogue où chacun tente de devenir un peu plus compréhensible à l'autre. Leur projet éthique s'oriente ainsi vers une éthique de l'hospitalité, d'autant plus construite et réfléchie qu'elle accueille les plus lointains : les Voyageurs, les enfants déficients. Selon le principe de l'hospitalité, chacun sait que l'opacité de l'autre est la condition fondatrice de l'altérité, et qu'il doit faire l'effort du dialogue, du partage langagier des expériences et de leur mise à l'épreuve pour produire des savoirs nouveaux dans une forme de vie assumée collectivement.

Plus tardivement, la poursuite des recherches en direction des titulaires première année a permis d'identifier une troisième épreuve, celle de l'assomption de la responsabilité ${ }^{27}$. Du point de vue éthique, elle signifie que le débutant accepte de passer « de l'autre côté du miroir ${ }^{28}$ et qu'il prend en charge globalement le poids moral de son agir professionnel : l'acteur s'assume enfin comme agent moral. Il s'agit bien d'une épreuve, et non d'un simple passage, dans la mesure où son échec conduit le jeune enseignant à la démission à l'orée même de sa carrière. Il y a deux faces bien distinctes que décrivent les entretiens. Tout d'abord un moment réflexif, en fin de formation, qui amène le débutant à faire le bilan des compétences construites et généralement à douter de leur étendue. Mais ce doute est soumis lui-même à la pression de l'environnement professionnel et s'en trouve amplifié parfois jusqu'à la démesure : tous y participent, parents d'élèves, collègues sceptiques, conseillers pédagogiques. Or dès ce moment, il ne concerne plus seulement certaines compétences du débutant, mais attaque sa qualité même et son aptitude à enseigner.

Aussi la réflexion découvre-t-elle enfin le cœur du problème de la responsabilité morale de l'enseignant, qui n'est pas de posséder par avance les réponses à toutes les difficultés potentielles de l'acte pédagogique, mais, tout différemment, de comprendre que l'on a construit les compétences permettant de problématiser éthiquement sa praxis quelles que soient les circonstances à venir, parce que l'on sait s'y prendre avec autrui dans le but d'assurer son éducation. Le débutant assume sa responsabilité lorsqu'il reconnaît avec certitude qu'il peut avoir confiance dans la relation pédagogique, parce qu'il peut faire confiance dans l'intelligence herméneutique des élèves et que ceux-ci pourront avoir confiance dans la sienne propre : à aucun moment, les élèves et leur maître ne seront démunis et exposés à la perte de sens, au dépassement des limites, à la dissolution de la communauté, à

\footnotetext{
${ }^{26}$ Itard J. (1964) ; Mémoire et Rapport sur Victor de l'Aveyron. Paris, Bibliothèque 10-18, 1964.

${ }^{27}$ Moreau D. \& Lesterlin B. «L'assomption de la responsabilité : l'entrée des jeunes enseignants dans la professionnalité »; $8^{\text {ème }}$ Biennale, Lyon, 2006.

${ }^{28}$ Hughes E.C. Le regard sociologique, Paris, EHESS, 1992.
} 
cette catalysis si redoutée des Grecs. Cette assomption de la responsabilité manifeste des compétences qui peuvent s'exprimer à partir des principes herméneutiques premiers qui fondent l'éthicité de la relation éducative : le principe d'indulgence ${ }^{29}$, l'anticipation de la perfection $^{30}$, la pertinence interprétative.

Il est permis de comprendre que les compétences ainsi synthétisées par l'assomption de la responsabilité résultent des deux épreuves antérieurement décrites, et que dès lors ce troisième moment est une interprétation créatrice dans laquelle le débutant comprend, en un bloc, l'essence de sa professionnalité : son noyau éthique propre. Aussi pensons-nous qu'il devient possible désormais d'apporter des éléments de réponses à nos questions initiales.

\section{(Conclusion)}

\section{g) L'orientation de la formation : déontologie ou éthique appliquée ?}

Le problème qui était posé était celui de savoir si la requête d'une éthicité concrète des enseignants devait être satisfaite par l'imposition d'une déontologie institutionnellement déterminée ou s'il était pertinent de laisser les praticiens résoudre par eux-mêmes cette question. Il est légitime de penser que cette alternative n'est peut-être pas fondée. Nos recherches ont mis en évidence la genèse d'une éthique professionnelle, mais elles n'ont pas prétendu être une enquête statistique de masse, qui, au demeurant, n'eut été qu'un visage fugitif de la morale enseignante. La proposition que nous pouvons retenir est en effet conditionnelle : si les enseignants entrent dans une démarche de professionnalisation, alors celle-ci est centrée sur l'éthique et se structure selon les modes que nous avons décrits, comme les seuls pertinents a priori, étant données les conditions formelles du problème. Mais cette proposition est purement contrefactuelle, si on veut l'appliquer à des enseignants non professionnalisés : «s'ils s'étaient professionnalisés, ils auraient construit une éthique », et cependant à nos yeux elle est suffisante pour résoudre la difficulté, si on la situe bien dans son origine, c'est-à-dire en amont. En effet, un enseignant non professionnalisé est un praticien qui n'a pas construit les compétences de réflexivité, d'auto-évaluation, d'auto-formation, d'auto-efficacité personnelle et de communication entre pairs que l'on s'accorde à reconnaître actuellement $^{31}$. En un mot, un tel enseignant ne peut pas prendre la position d'un agent moral. Quels seraient les effets dans ce contexte d'une déontologie? Une véritable déontologie professionnelle (médecins, avocats, magistrats) se concrétise toujours par la prestation d'un serment. Par un tel acte, le praticien reconnaît publiquement sa réflexivité comme constitutive de sa posture professionnelle: manquer à son serment, c'est manquer à la promesse publiquement faite d'agir selon les principes éthiques qui permettent de problématiser les situations professionnelles, et de réguler ses pratiques selon ces normes. Une déontologie ne crée pas la réflexivité, puisqu'elle la suppose. Aussi s'avère-t-elle éthiquement inutile dans l'hypothèse où celle-ci est en défaut, et ne peut être confondue avec des injonctions de moralité qui ne donnent, comme le montre Kant, qu'une conformité extérieure aux lois. En ce sens, l'idée même d'un Code, si elle peut interdire des actes en prévoyant des sanctions, ne peut en aucun cas favoriser un gain en éthicité concrète. Dans l'éducation, le droit positif suffit à réprimer les actes illégaux, mais aucun code ne peut bannir les pratiques illégitimes qui ne respectent pas la personnalité morale de l'élève, son droit à l'éducation qui inclut d'être accueilli avec bienveillance dans une structure d'éducation.

\footnotetext{
${ }^{29}$ Quine W. O. Le mot et la chose, Paris, Flammarion, 1977.

${ }^{30}$ Gadamer H. G. op. cit.

${ }^{31}$ Altet M. La formation professionnelle des enseignants, Paris, PUF, 1994.
} 
Il faut cependant distinguer entre deux requêtes en vue d'une déontologie qui pourrait être qualifiée de «forte» ou de «faible». La première, posée par G. Longhi ${ }^{32}$ se heurte à deux arguments puissants. Le premier est que sa construction prend la figure d'une morale éclectique, qui, comme l'avait analysé V. Brochard ${ }^{33}$ à propos de la morale scolaire « rassemble des éléments épars, des fragments de doctrines pris de tous côtés et rapprochés assez arbitrairement ». L'éclectisme moral, comme le montre J. Vuillemin, interdit toute cohérence éthique, et l'on ne comprend pas comment elle pourrait aider les professionnels. Le second argument est qu'un Code, comme on l'a dit, interdit le problème de l'Application et légitimise un certain fondamentalisme qui séduit parfois de jeunes enseignants du secondaire : les réponses morales sont connues avant que les problèmes ne soient posés, et les décisions sont disponibles en dehors de toute compréhension des situations.

Contre ces dangers, Eirick Prairat défend l'idée d'une déontologie « faible ${ }^{34} »$ :

«Il convient donc moins de proposer un code qu'une charte, moins un catalogue d'interdits et de prescriptions qu'un texte plus ramassé et articulé autour de quelques articles clefs ». Si cette proposition conserve la responsabilité portée par l'accès à la question de l'application, nous pensons cependant que son écriture devrait être a posteriori le résultat de la formation professionnelle, plutôt que le préalable d'un agir d'enseignant. Ce serait l'introduction d'un travail d'explicitation autoréférentielle à partir des principes universels sous-tendus par l'acte d'éduquer, entrepris par les enseignants débutants dans l'analyse herméneutique de leur pratiques professionnelles ${ }^{35}$ au cours de leur formation et de leur entrée dans le métier.

L'éthicité, par définition même, résulte d'une décision libre du sujet d'engager sa pensée et ses actes dans la perspective du choix moral, telle que l'avait ouverte la question socratique : «comment dois-je vivre, pour être le mieux possible un homme ? » elle ne peut donc être contrainte. C'est pourquoi il faut se tourner à nouveau vers l'éducation, qui est la réponse platonicienne à la question, ici, à l'éducation de soi-même, telle que la formation la rend possible. C'est en effet dans la formation professionnelle que se joue la question de la genèse de l'éthique des enseignants. Nous avons mis en évidence chez les formateurs cette compétence à l'étayage de la réflexion morale du débutant, très loin en général de la modélisation du compagnonnage propre aux métiers. Les jeunes débutants initient eux-mêmes cet étayage par une demande forte d'un partage de la problématisation dans la structure de l'accompagnement, et se montrent extrêmement réticents à toute tentative d'inculcation de valeurs - c'est une conséquence de la vulnérabilité. Les pratiques de formation qui leur paraissent alors les plus adéquates sont l'accompagnement proche et l'analyse des pratiques professionnelles, conduites dans un souci herméneutique. Aussi nos conclusions vont-elles, à contre-courant d'une tendance actuelle, vers un optimisme raisonné dans les compétences des acteurs de l'éducation eux-mêmes à développer la dimension éthique de leur profession, dans la mesure où la formation qu'ils ont reçue leur permet d'accéder à la problématisation, seule source légitime d'une éthique appliquée, et de refuser les fondamentalismes moraux portés par des convictions monologiques sans exercice de la responsabilité. En tant qu'ouverture sur les Neö̈, les «nouveaux venus », l'éducation construit chemin faisant, par ses pratiques, les normes qui leur permettent de prendre place dans le monde.

\footnotetext{
32 Longhi G. Pour une déontologie de l'enseignement, Paris, ESF, 1998.

${ }^{33}$ Brochard V. La morale éclectique, in : Etudes de philosophie ancienne et moderne, Paris, Vrin, 1974.

${ }^{34}$ Prairat E. De la déontologie enseignante, Paris, PUF 2005.

${ }^{35}$ C'est la fonction des séances de formation par l'analyse herméneutique des pratiques, menées par exemple à l'IUFM de Nantes, et à notre avis également la visée des travaux de l'équipe d'Anne Jorro à l'Université de Toulouse II.
} 